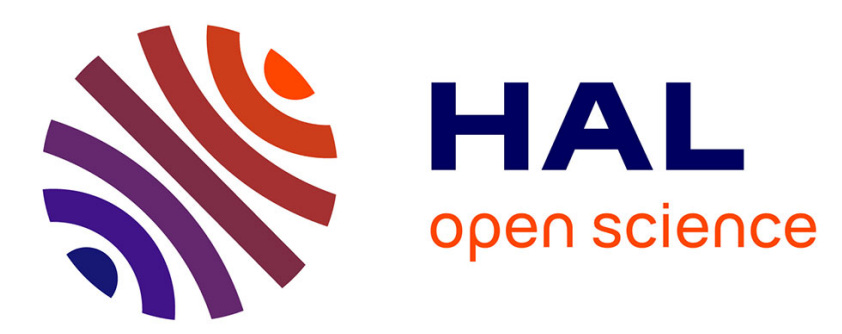

\title{
Characterisation of in-vivo mechanical action of knee braces regarding their anti-drawer effect
}

\author{
B. Pierrat, R. Oullion, J. Molimard, L. Navarro, M. Combreas, S. Avril, R. \\ Philippot, Paul Calmels
}

\section{- To cite this version:}

B. Pierrat, R. Oullion, J. Molimard, L. Navarro, M. Combreas, et al.. Characterisation of in-vivo mechanical action of knee braces regarding their anti-drawer effect. The Knee, 2015, 22 (2), pp.80-87. 10.1016/j.knee.2014.12.001 . hal-01137491

\section{HAL Id: hal-01137491 \\ https://hal.science/hal-01137491}

Submitted on 30 Mar 2015

HAL is a multi-disciplinary open access archive for the deposit and dissemination of scientific research documents, whether they are published or not. The documents may come from teaching and research institutions in France or abroad, or from public or private research centers.
L'archive ouverte pluridisciplinaire HAL, est destinée au dépôt et à la diffusion de documents scientifiques de niveau recherche, publiés ou non, émanant des établissements d'enseignement et de recherche français ou étrangers, des laboratoires publics ou privés. 


\title{
Characterisation of in-vivo mechanical action of knee braces regarding their anti-drawer effect
}

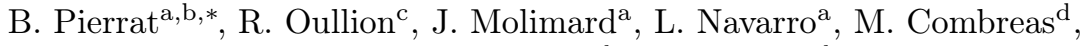 \\ S. Avril ${ }^{\mathrm{a}}$, R. Philippot ${ }^{\mathrm{d}}$, P. Calmels ${ }^{\mathrm{c}, \mathrm{d}}$ \\ ${ }^{a}$ Ecole Nationale Supérieure des Mines, CIS-EMSE, CNRS:UMR5307, LGF, F-42023 \\ Saint-Etienne, France \\ ${ }^{b}$ Pôle des Technologies Médicales, F-42000 Saint-Etienne, France \\ ${ }^{c}$ Department of Clinical Physiology of Exercise, Units of Sport Medicine and Myology, \\ Bellevue Hospital, University Hospital Center, Saint-Etienne, France \\ ${ }^{d}$ Orthopaedic surgery department, North Hospital, 42055 Saint Etienne, Cedex 2, France
}

\section{Introduction}

The knee is the largest joint in the body and supports high loads, up to several times the body weight. It is vulnerable to injury during sport or professional activities, potentially leading to chronic knee instability. This instability is a functional issue to the patient and is characterized by a "wobbly" feeling. Different internal structures take part in joint stabilization by passive (ligaments, capsule) or active (neuro-muscular system and proprioception) action. The most common injury involves the anterior cruciate ligament (ACL) complete or partial rupture: it is involved in $24 \%$ of all knee injuries and $59 \%$ of ligamentous injuries [1]. In the United States, the annual incidence in the general population is approximately 1 in 3500 with 100,000 ACL reconstructions performed each year $[2,3]$. These conditions are a huge burden on individuals and healthcare systems.

Diagnosis of knee instability involves a discussion with the patient and a clinical examination, usually the Lachman test. It consists in a manual anterior translation of the tibia aiming at putting the ACL in tension. By practice, the examiner is able to grade the laxity by severity [4]. However, its sensitivity and specificity to detect complete ACL ruptures depends on the experience of the examiner, the patient's body type and the delay between the accident and examination [5]. In order to reduce this variability, arthrometers were developed. These devices apply an increasing force to induce a postero-anterior drawer and measure the corresponding translation. Displacement-load curves of the healthy and injured knees are compared; ACL rupture is ascertained when differential laxity is higher than a certain threshold. Well-known arthrometer devices are the KT-1000 developed by [6] and the GNRB ${ }^{\circledR}[7]$. The former is very popular

${ }^{*}$ Corresponding author. E-mail: baptiste.pierrat@gmail.com. Phone: +33641732883. 
and has been widely studied; its sensitivity to detect complete ACL ruptures is $77 \%$ and its specificity $90 \%$ (threshold: differential laxity of $3 \mathrm{~mm}$ at $130 \mathrm{~N}$ ). The latter was developed recently; two studies $[5,7]$ highlighted the superiority of this device compared to similar apparatus. A differential threshold of $3 \mathrm{~mm}$ at $134 \mathrm{~N}$ is used to determine complete rupture: its sensitivity is $70 \%$ and specificity $99 \%$. This arthrometer device is also used to diagnose partial ruptures (threshold of $1.5 \mathrm{~mm}$ at $134 \mathrm{~N}$ ) with a sensitivity of $80 \%$ and a specificity of $87 \%$.

Knee braces or orthoses are usually part of the standard therapy for knee instability and are commonly prescribed by physicians and medical practitioners. Their claimed mechanical effects are to support/align the joint and increase proprioceptive input [8]. However, very few studies actually show significant actions, from biomechanical studies to therapeutic trials [9, 10]. Mechan$\mathrm{ical} /$ physiological effects have been emphasized, but the mechanisms of action have been poorly characterized $[8,9,10,11,12,13]$. What is more, subjective evaluations of patients highlight a large demand for these products; therefore, their efficiency is still widely discussed among medical experts. In particular, the relative importance of the two principal stabilizing mechanisms is not known:

- joint stiffening by adding supporting structures, e.g. hinged bars secured to the joint by straps and fabric (passive mechanism)

- neuromuscular control enhancement by proprioceptive effect (active mechanism)

As a consequence of these uncertainties, medical practitioners and manufacturers still lack a simple evaluation tool for knee orthoses. A French committee of experts highlighted this problem [14] and stated that orthoses must be evaluated by taking both the mechanisms of action and the desired therapeutic effects into account.

Although the passive action of knee braces to prevent a drawer motion has already been characterized on surrogate limbs [15, 16, 17], only one attempt has been made to link these measures to corresponding expected in-vivo actions through numerical modelling [18, 19], highlighting a passive stiffening much lower than what is brought by intact ligaments. However, a real clinical study is still needed to validate the numerical results and investigate the influence of patient-specific factors such as morphology, injury severity, neuromuscular adaptation and instability feeling control.

This study is aimed at objectively quantifying the joint stiffening action of various commercial braces in-vivo using a GNRB ${ }^{\circledR}$ arthrometer on a number of pathological patients.

\section{Material and methods}

The described protocol has been validated by the Ethical Committee of the University Hospital of Saint-Etienne, France. 


\subsection{Patients}

A sample of 25 subjects (16 males and 9 females) were selected for this study among hospitalized patients in the orthopædic and traumatology surgery unit of the University Hospital of Saint-Etienne, France. The inclusion criteria were the following:

- Patients being treated for functional knee instability, either a chronic laxity or after ACL injury, before reconstructive surgery.

- Acute knee anterior instability (positive Lachman drawer test).

- Patients that had been prescribed an arthrometer test in this context.

- Independent walkers.

- Patients who have agreed study conditions and signed the information notes.

Some exclusion criteria were also identified:

- Very recent trauma (e.g. acute sprained knee).

- Other musculoskeletal disease (e.g. fracture, arthrosis...).

Subjects were prospectively and consecutively recruited from February 2013 to July 2013. The characteristics of the whole recruited population are described in Table 1.

\subsection{Knee braces}

Three local orthotic manufacturers agreed to lend their products for this

study (Gibaud ${ }^{\circledR}$, Lohmann \& Rauscher ${ }^{\circledR}$ and Thuasne ${ }^{\circledR}$ ). As a wide range of knee braces is available (both custom-fitted and off-the-shelf orthoses), the focus was placed on fabric-based off-the-shelf hinged braces which are the highestselling products of these manufacturers for the studied clinical indication.

The following products were selected for testing (one control brace and one hinged brace for each manufacturer):

- Compression sleeve: Thuasne Genuaction (control brace).

- Fabric hinged orthoses:

- Gibaud GebuGib Stab

- Lohmann \& Rauscher Ligaction Pro

- Thuasne Ligaflex Evolution

They are depicted in Figure 1.

From now on, the names of the braces have been replaced by numbers to de-identify the products and not penalize the manufacturers. 


\begin{tabular}{|c|c|c|c|c|c|c|c|}
\hline ID & Sex & Age & $\begin{array}{l}\text { Height } \\
\text { (cm) }\end{array}$ & $\begin{array}{l}\text { Weight } \\
\text { (kg) }\end{array}$ & $\begin{array}{l}\text { Knee circ. } \\
\quad(\mathrm{cm})\end{array}$ & $\begin{array}{c}\text { Laxity at } \\
134 \mathrm{~N}, \\
\text { healthy } \\
\text { knee }(\mathrm{mm})\end{array}$ & $\begin{array}{c}\text { Laxity at } \\
134 \mathrm{~N}, \\
\text { pathol. } \\
\text { knee }(\mathrm{mm})\end{array}$ \\
\hline $1^{*}$ & M & 26 & 184 & 98 & 39 & 2 & 5.2 \\
\hline 2 & $\mathrm{~F}$ & 21 & 159 & 54 & 35 & 4.4 & 6.2 \\
\hline $3^{*}$ & $\mathrm{M}$ & 44 & 177 & 75 & 38 & 4.8 & 6.5 \\
\hline 4 & $\mathrm{M}$ & 20 & 182 & 80 & 39 & 4.4 & 7.2 \\
\hline $5^{*}$ & $\mathrm{~F}$ & 26 & 167 & 53 & 33 & 3.5 & 4.4 \\
\hline 6 & $\mathrm{M}$ & 26 & 167 & 60 & 35 & 3.9 & 7.5 \\
\hline 7 & $\mathrm{~F}$ & 17 & 163 & 57 & 37 & 4 & 9.2 \\
\hline 8 & $\mathrm{M}$ & 28 & 174 & 68 & 35 & 5 & 7.8 \\
\hline 9 & $\mathrm{~F}$ & 34 & 169 & 69 & 40 & 4.7 & 10.1 \\
\hline 10 & M & 20 & 173 & 78 & 37 & 3.6 & 5.9 \\
\hline 11 & $\mathrm{~F}$ & 29 & 171 & 56 & 36 & 4.8 & 8.5 \\
\hline 12 & $\mathrm{M}$ & 22 & 184 & 75 & 40 & 5.2 & 8.2 \\
\hline 13 & $\mathrm{M}$ & 23 & 178 & 63 & 37 & 3.8 & 8.3 \\
\hline 14 & $\mathrm{M}$ & 25 & 184 & 96 & 43 & 4.9 & 8.2 \\
\hline $15^{*}$ & $\mathrm{M}$ & 28 & 175 & 65 & 36 & 7.6 & 8.8 \\
\hline $16^{*}$ & $\mathrm{M}$ & 15 & 180 & 65 & 38 & 4.8 & 13.5 \\
\hline $17^{*}$ & $\mathrm{M}$ & 13 & 171 & 56 & 36 & 5.8 & 11 \\
\hline 18 & $\mathrm{M}$ & 24 & 183 & 92 & 42 & 4.7 & 7.4 \\
\hline 19 & $\mathrm{~F}$ & 43 & 164 & 74 & 37 & 4.3 & 6.2 \\
\hline 20 & $\mathrm{M}$ & 18 & 175 & 72 & 42 & 6.3 & 9.3 \\
\hline $21^{*}$ & $\mathrm{~F}$ & 26 & 160 & 61 & 37 & 3.6 & 11.5 \\
\hline $22^{*}$ & $\mathrm{M}$ & 48 & 159 & 88 & 41 & 3.4 & 4.9 \\
\hline $23^{*}$ & $\mathrm{~F}$ & 59 & 165 & 70 & 42 & 4.4 & 5.7 \\
\hline 24 & $\mathrm{M}$ & 29 & 182 & 76 & 39 & 5.1 & 10.2 \\
\hline 25 & $\mathrm{~F}$ & 18 & 169 & 56 & 34 & 4.4 & 6.6 \\
\hline av. & & 27 & 173 & 70 & 38 & 4.5 & 7.9 \\
\hline
\end{tabular}

Table 1: Subject characteristics with measured laxities of healthy and pathological knees using the GNRB ${ }^{\circledR}$ arthrometer. * Subjects not included in the final results due to outlying GNRB ${ }^{\circledR}$ measurements.

The compression sleeve was selected to act as a control brace, because its passive mechanical stiffening action was characterised as negligible [18]. Consequently, its effect should not be measured by the arthrometer unless its proprioceptive effect activates muscular stiffening during the tests. The three other braces are similar in design (fabric with hinged rigid bars and straps) but previous work showed slightly different levels of mechanical actions [18]. They are prescribed for moderate and serious sprains, chronic laxity or during the rehabilitation process. It is noteworthy that brace $\# 2$ slightly differs from the two other braces in the sense that it features supplementary helical straps, and because it had an open design (unlike the two others that were closed cylinders).

Braces were available in different sizes and chosen accordingly to the subject's knee circumference. They were adjusted by the subjects themselves. 


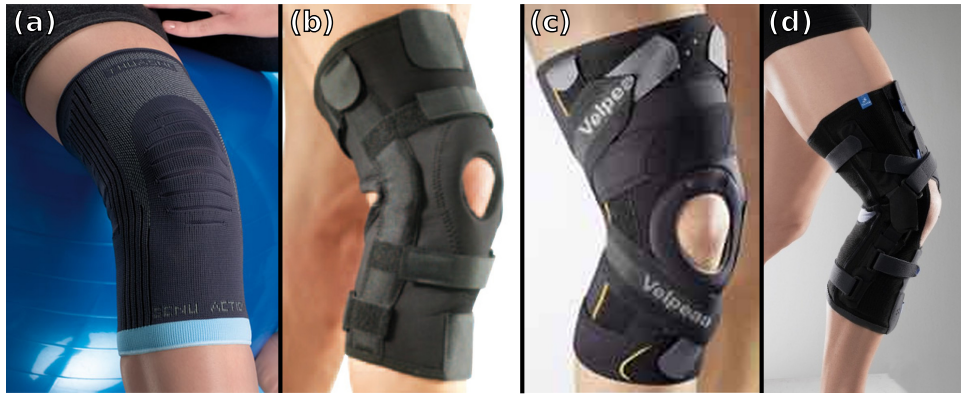

Figure 1: Panel of knee braces selected to be tested: Thuasne Genuaction (a), Gibaud GenuGib Stab (b), Lohman \& Rauscher Ligaction Pro (c) and Thuasne Ligaflex (d).

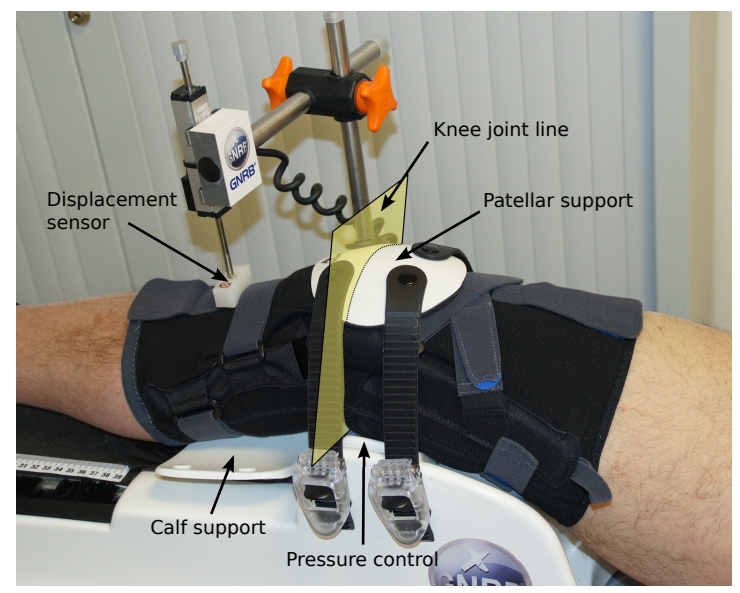

Figure 2: GNRB ${ }^{\circledR}$ system.

\section{3. $G N R B^{\circledR}$ arthrometer}

The GNRB ${ }^{\circledR}$ system is shown in Figure 2. It has already been described and evaluated in the literature $[5,7,20]$.

The usual test protocol is the following. The patient is lying on a table; the lower limb is placed in the support such as the knee is in neutral rotation and the joint line is aligned between the calf support and the thigh support. The ankle and patella are held in place by strapped supports. The tightening of the latter support is controlled by a force sensor to be about $60 \mathrm{~N}$. The patient is asked to relax and an electric actuator exerts an increasing load (up to $250 \mathrm{~N}$ ) at $11 \mathrm{~mm} / \mathrm{s}$ through the calf support. A displacement transducer (accuracy of $0.1 \mathrm{~mm}$ ) measures the relative displacement of the tibia with respect to the patella with a sampling of $5 \mathrm{~N}$, and a displacement-force curve is plotted. This procedure is usually performed once to check if the knee is painful, then three times in a row to check the reproducibility and compute the final response as 


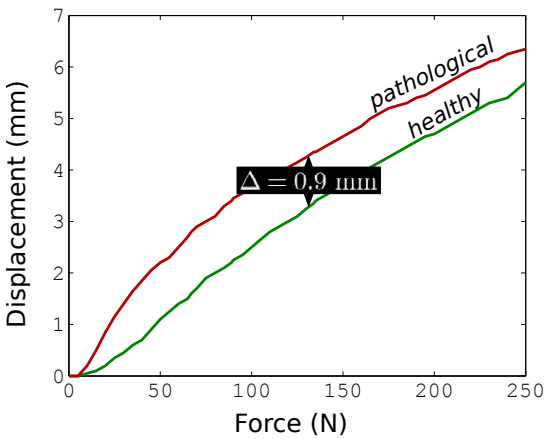

(a) Low grade injury

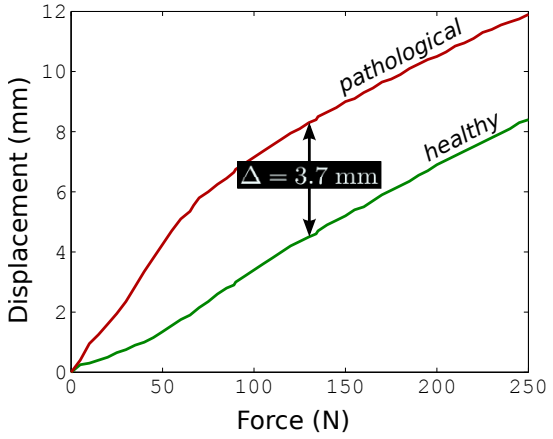

(b) Complete ACL rupture

Figure 3: Response curves as given by the GNRB $^{\circledR}$ device: (a) partial tear with differential laxity of $0.9 \mathrm{~mm}$ at $134 \mathrm{~N}$ (subject 5); (b) complete ACL rupture with differential laxity of $3.7 \mathrm{~mm}$ at $134 \mathrm{~N}$ (subject 11 ).

the mean of these three curves. The maximum force is usually $250 \mathrm{~N}$ but may be reduced to 200 or $134 \mathrm{~N}$ if the patient feels pain. The usual protocol consists in testing both the healthy and pathological knee to compare the responses and diagnose the degree of laxity. The criterion for the diagnosis of ligament injury is the differential laxity $\Delta$ at $134 \mathrm{~N}$ : complete ACL rupture is characterised by $\Delta \geq 3 \mathrm{~mm}$ (sensitivity of $70 \%$ and specificity of $99 \%$ [7]) and partial rupture by $\Delta \geq 1.5 \mathrm{~mm}$ (sensitivity of $80 \%$ and specificity $87 \%[7]$ ).

Examples of curves given by this usual testing protocol for subjects 5 and 11 are reported in Figure 3, showing two different grades of injury: a partial tear and a complete ACL rupture.

Preliminary tests have showed no difficulty to perform laxity measurements on this apparatus with any of the knee braces because they all feature a patella opening allowing the patellar support to push directly on this area, and the rigid elements of the braces were not interacting with the securing system of the GNRB $^{\circledR}$ (the hinged bars were free to move, as seen in Figure 2).

\subsection{Protocol}

As the normal diagnosis for these patients involved laxity measurement of both healthy and pathological knee with the GNRB ${ }^{\circledR}$ arthrometer, they were asked to take part of this study and perform 4 supplementary tests with the four different knee braces fitted on their pathological knee. Patients were also asked to grade the braces using a Visual Analog Scale (VAS) regarding two subjective criteria: stabilization sensation (0: no stabilization; 10: complete stabilization) and comfort (0: intolerable; 10: extremely comfortable).

As the internal structures of the knee were reported to have a highly viscoelactic behaviour [21], one may expect that the repetition of tests would influence the measured laxities. Consequently, the order of the 4 braces to be tested was randomized for each subject.

The testing protocol took place in the following manner: 
1. The usual laxity measurements were performed (on both healthy and pathological knees), with maximum forces of $250 \mathrm{~N}$ if possible, or lower if the subject experienced pain.

2. The appropriate size of the tested brace was fitted and adjusted by the patient himself, who had to walk around the room to be sure that it was properly tightened and fitted. Special care was taken in aligning the axis of rotation of the hinged bars with the axis of rotation of the knee.

3. The subjective evaluation of stabilization and comfort was performed after a few movements: $20 \mathrm{~m}$ walking, one-foot standing and squatting.

4. The subject was asked to place the braced pathological knee on the GNRB ${ }^{\circledR}$ for three consecutive tests (with maximum forces of $250 \mathrm{~N}$ or lower).

Steps 2 to 4 were repeated for each of the 4 braces.

A physical therapist experienced in using the $\mathrm{GNRB}^{\circledR}$ and in evaluating ACL deficiency performed the procedures.

\subsection{Data processing}

Collected data for each subject consisted in variables reported in Table 1, one averaged GNRB ${ }^{\circledR}$ test curve for the healthy knee and one for the pathological knee, three test curves for each brace and the two VAS scores for comfort and stabilization. The healthy knee was determined to be normal by subjective and objective testing and was used as the control.

Concerning laxity measurements, some patients were excluded from the final data due to outlying measurements:

- Patients with a differential laxity at $134 \mathrm{~N}$ lower than $1.8 \mathrm{~mm}$ (5 subjects) or higher than $7 \mathrm{~mm}$ (2 subjects).

- Patients who experienced pain and could not reach $200 \mathrm{~N}$ (1 subject).

- Patients who exhibited test curves with a highly different curvature than others (1 subject).

Consequently, data processing of laxity measurements was performed for 16 subjects (10 males and 6 females).

In order to compare the stiffening levels of the 4 braces, their action has to be decoupled from the inherent stiffness of the knee. For this purpose, the mechanical system could be modelled as three springs in parallel, as depicted in Figure 4: the ACL, the other structures of the knee and the brace. This parallel representation is valid because the displacement of the ensemble of structures is their common displacement. Consequently, the force applied on the ensemble is the sum of their individual forces.

As the arthrometer measures displacements for every $5 \mathrm{~N}$ load and produces displacement-load curves, the curves were interpolated using splines and resampled in displacement (every $0.05 \mathrm{~mm}$ ) to obtain load-displacement curves. This was done to be able to add or subtract the different load responses. Confidence intervals (CIs) on the means were computed using Student's $t$ distribution assuming that values were normally distributed. 


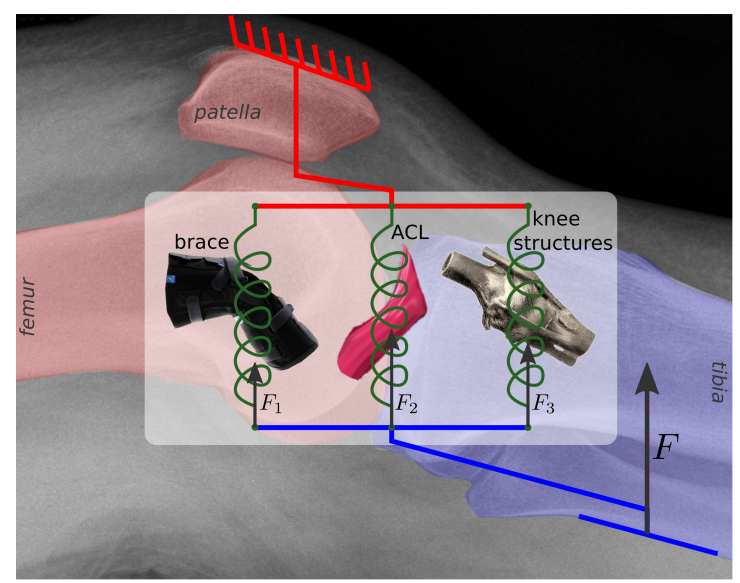

Figure 4: Schematic illustration of the braced knee mechanical system as three springs in parallel, representing the stiffness brought by the brace, the ACL and the other knee structures. In this case, the total force $F$ applied to the system is distributed among the springs so that $F=F_{1}+F_{2}+F_{3}$.

It was assumed that injured patients had a deficient ACL. Consequently, the following operations were performed for each subject to decouple the responses:

- Contribution of other knee structures: pathological knee curve.

- Contribution of the brace: braced pathological knee curve - pathological knee curve.

- Contribution of the ACL: healthy knee curve - pathological knee curve.

For comparing datasets between braces (VAS scores, brace effects), multiple comparison tests were performed. Outliers were defined as values larger than $q_{3}+\left(q_{3}-q_{1}\right)$ or smaller than $q_{1}-\left(q_{3}-q_{1}\right)$, where $q_{1}$ and $q_{3}$ are the 25 th and 75 th percentiles, respectively. This corresponds to approximately $\pm 2 \sigma$ and $95 \%$ coverage if the data are normally distributed. The Matlab ${ }^{\circledR}$ R2012 was used to process all data.

Finally, the overall additional stiffness brought by the braces was characterised in terms of $k$ indexes, as introduced in previous works $[18,19]$. This was performed by fitting a 1st order polynomial to the response of the braces for each subject, for displacements between 1 and $5 \mathrm{~mm}$ (linear part). The $k$ index is the slope of the fitted line.

\section{Results}

\subsection{Subjective evaluation of stabilization and comfort}

The results of the subjective evaluation of the stabilization and comfort sensation of the tested braces is shown in Figure 5. No statistical difference was 


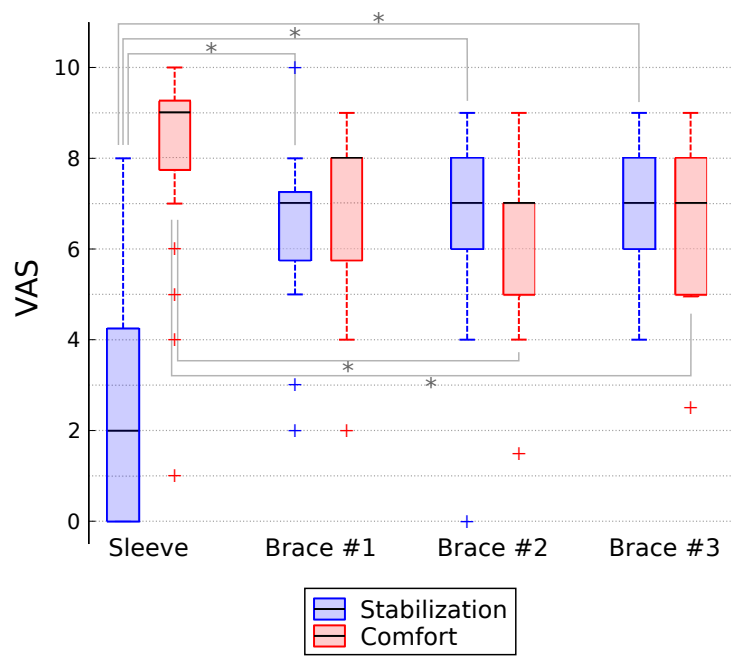

Figure 5: Box plot of the VAS score of the subjective evaluation of stabilization sensation and comfort of the different braces (25 subjects). On each box, the central black mark is the median, the edges of the box are the 25 th and 75 th percentiles and the whiskers extend to the most extreme data points not considered outliers. Stars show statistically different means between braces $(p \geq 0.95)$ and plus signs the outliers.

observed between the three hinged braces. However, the compression sleeve was reported to be statistically less stabilizing than the three braces, and more comfortable than braces $\# 2$ and $\# 3$. In all cases, a substantial scattering of the scores was observed.

\subsection{Healthy and pathological knees}

Raw test curves of the healthy and pathological knees as given by the GNRB ${ }^{\circledR}$ arthrometer are shown in Figure 6 for the 16 subjects. A high scattering of the curves can be noted. It was attempted to normalize GNRB ${ }^{\circledR}$ measurements by weight, size or knee circumference, but it did not affect the relative standard deviations. Besides, no significant statistical difference was observed between males and females. However, means of healthy and pathological knees were still statistically very different, as indicated by computing the average differential laxities: $3.3 \mathrm{~mm}$ at $134 \mathrm{~N}$ and $3.8 \mathrm{~mm}$ at $250 \mathrm{~N}$.

\subsection{Intra- and inter-subject variability}

Laxity tests with the braced pathological knees were performed three times and results were extracted before being averaged by the GNRB ${ }^{\circledR}$ system, so it was possible to investigate intra-subject variability of these data. It was calculated as the standard deviations of the displacement for the four different braces (the type of brace had no effect on the scattering of the results) at $134 \mathrm{~N}$ and $250 \mathrm{~N}$. A mean standard deviation of $2.3 \mathrm{~mm}$ was found at $134 \mathrm{~N}$ and 


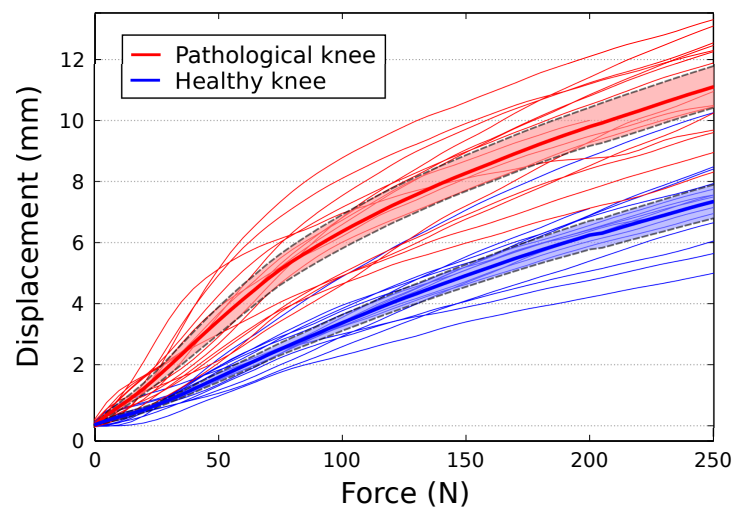

Figure 6: Displacement-load test curves given by the GNRB ${ }^{\circledR}$ arthrometer of healthy and pathological knees for the 16 subjects. Bold lines represent the means and dashed lines the $95 \%$ CIs for the means.

$4.2 \mathrm{~mm}$ at $250 \mathrm{~N}$. Both these values corresponded to a relative dispersion of $3.8 \%$, which indicated a good reproducibility of the tests for each patient.

Inter-subject variability was characterised as the standard deviation between averaged measurement. A mean standard deviation of $15 \mathrm{~mm}$ was found at $134 \mathrm{~N}$ and $19 \mathrm{~mm}$ at $250 \mathrm{~N}$ (relative dispersions of $24 \%$ and $17 \%$ respectively). This high variability shows that GNRB ${ }^{\circledR}$ measurements are very specific to each subject; this observation may be explained by different grades of injury and by the documented variability in mechanical properties of internal structures between individuals [22]. It highlights the importance of statistical tests to determine if further results are significant for this number of subjects.

\subsection{Decoupled contributions of the structures}

The mean decoupled contributions of the different structures computed as described in Section 1.5 is shown in Figure 7. The healthy knee response is also shown to have an idea of the relative contribution of each structure. Firstly, the stiffness brought by the compression sleeve was very small, although it was not zero. The load taken by this sleeve increased between 0 and $1 \mathrm{~mm}$ drawer and rapidly stabilized around $14 \mathrm{~N}$ for higher displacements. The three hinged braces have relatively different responses and may be graded in term of antidrawer efficiency using these measurements: at $5 \mathrm{~mm}$ displacement, reaction forces were 27,37 and $53 \mathrm{~N}$ for brace $\# 3, \# 1$ and $\# 2$ respectively. The curve profiles were similar with a rapid load increase up to $1 \mathrm{~mm}$ drawer, followed by a slower increase. It is noteworthy that reaction forces peaked around $5 \mathrm{~mm}$ displacement and slightly decreased thereafter for braces $\# 3$ and $\# 1$. This was not characteristic of brace $\# 2$, although this particularity may be explained by the fact that no high displacements were measured with this brace, so this curve stops before the other ones. 


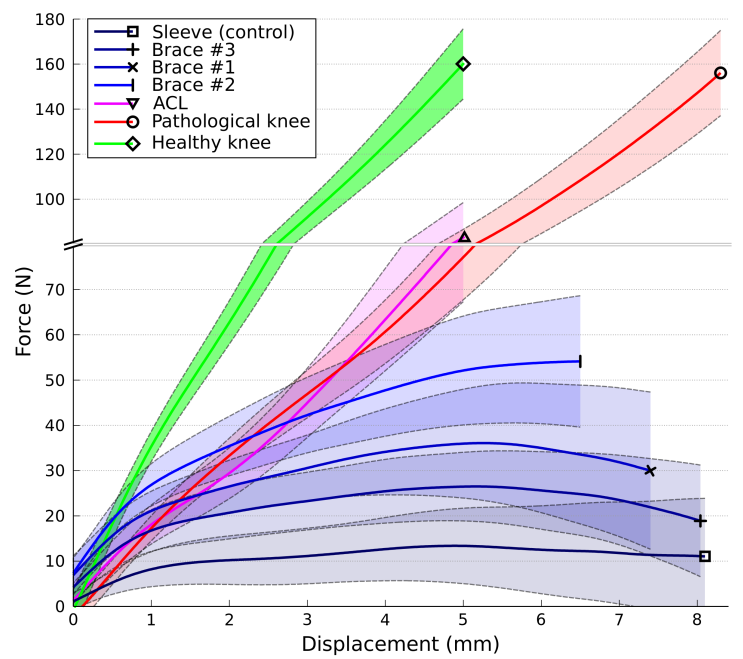

Figure 7: Mean decoupled load-displacement curves of the following structures for the 16 subjects: the compression sleeve, the three hinged braces, the ACL, the other structures of the knee and the healthy knee (as reference). Bold lines represent the means and dashed lines the $95 \%$ CIs for the means.

Confidence intervals were large and some overlaps are visible, meaning that the responses between some braces were not statistically different $(p \geq 0.95)$. Only the compression sleeve had a significantly different response for displacements lower than $4 \mathrm{~mm}$.

The load-displacement curve of the ligament exhibited a different profile: its response was more linear, even slightly exponential. Consequently, forces at low displacements were lower than values measured for braces, then largely exceeded these devices for higher displacements, reaching $83 \mathrm{~N}$ at $5 \mathrm{~mm}$. This overrun occurred around $0.8 \mathrm{~mm}$ for brace $\# 3,1.6 \mathrm{~mm}$ for brace $\# 1$ and $2.8 \mathrm{~mm}$ for brace \#2.

Interestingly, the mean response of the other structures of the knee (obtained with the pathological knees) was found to be very close to the response of the ACL alone, even if the ACL curve was concave while the other curve had a convex profile. This means that the ACL contributed to about half of the overall knee stiffness between 0 and $5 \mathrm{~mm}$ anterior displacement.

When comparing the healthy knee responses to the braced pathological knees, it was observed that the braces brought a substantial amount of stiffness compared to the pathological joints. In average, the knees braced with devices $\# 3, \# 1$ and $\# 2$ applied an even greater reaction force than the healthy joints for anterior displacements below $0.8 \mathrm{~mm}, 1.6 \mathrm{~mm}$ and $2.8 \mathrm{~mm}$ respectively. At $5 \mathrm{~mm}$ displacement, the relative load contributions normalized to the average healthy knee were the following (with $95 \%$ CI on the means):

- Pathological knee alone: $48 \pm 6 \%$ of the healthy knee. 
- Pathological knee braced with compression sleeve: $57 \pm 8 \%$ of the healthy knee.

- Pathological knee braced with brace \#3: $65 \pm 7 \%$ of the healthy knee.

- Pathological knee braced with brace \#1: $71 \pm 8 \%$ of the healthy knee.

- Pathological knee braced with brace \#2: $81 \pm 9 \%$ of the healthy knee.

\subsection{Structure effects computed as $k$ indexes.}

As the responses were rather linear within the regression domain $(1-5 \mathrm{~mm})$, the fits were good and the coefficients of determination $R^{2}$ were almost always higher than 0.9. The mean $k$ indexes of the different structures are displayed in Table 2. The ranking of the different devices in terms of efficiency to prevent drawer is the same as previously presented: the compression sleeve exhibited a very low mechanical action, and hinged braces presented large differences. As the standard deviations were high, the $p$-values were computed for each pair to determine whether differences were significant $(p \leq 0.05)$. Each pair was significantly different except the following structures: braces $\# 1$ and \#2 $(p=0.27)$, brace $\# 3$ and compression sleeve $(p=0.58)$ and ACL and pathological knee $(p=0.75)$. Braces $\# 1$ and $\# 2$ were found to bring significantly more stiffness than a simple sleeve. However, the $k$ index of the ACL was found to be twice higher than the index of the most efficient brace. The results did not allow to determine a significant difference between braces $\# 1$ and $\# 3$, and between braces \#1 and \#2.

\begin{tabular}{lccc}
\hline Structure & $\begin{array}{c}\text { Mean } k \text { index } \\
(\mathrm{N} / \mathrm{mm})\end{array}$ & $\begin{array}{c}\text { Standard deviation } \\
(\mathrm{N} / \mathrm{mm})\end{array}$ & $\mathrm{N}$ \\
\hline Compression sleeve & 1.2 & 3.0 & 15 \\
Brace \#1 & 5.5 & 2.8 & 14 \\
Brace \#2 & 7.1 & 4.3 & 15 \\
Brace \#3 & 2.0 & 2.5 & 15 \\
ACL & 13.9 & 3.6 & 12 \\
Pathological knee & 14.3 & 4.8 & 16 \\
Healthy knee & 28.3 & 5.1 & 14 \\
\hline
\end{tabular}

Table 2: Mean $k$ indexes computed for the different structures of $\mathrm{N}$ subjects after removing the outliers.

Finally, it is noteworthy that no correlation was found between the differential laxity and the $k$ indexes of the braces, meaning that the devices had the same mechanical effect for various degrees of laxity. Subsequently, no device was found to act differently between males and females, and no correlation was found with age or knee circumference. Note that this may only mean that the number of subjects was not high enough to determine significant correlations. 


\section{Discussion}

This work was preceded by two other studies that introduced numerical [19] and experimental [18] tools to evaluate knee orthoses with a similar approach, so these studies take part in the discussions. Some limitations of the presented methodology have also been identified and are discussed below, but they were taken into account and do not discredit the given results.

Most of the recruited subjects did not usually wear a knee brace. Consequently, they may have adjusted their devices in a different manner as if they were regularly wearing them, and this fact must be taken into account when looking at the VAS scores. It may partly explain the high scattering of the grades, as the subjects did not have a common reference sensation of stabilization and comfort of braces.

No significant relationship was found between subjective stabilization grades and objective stiffening measurements for the 3 different hinged braces. It means that subjects were not able to grade the efficiency of these devices based on their feelings, and that physicians and manufacturers should rely on objective evaluation rather than on patient's feelings about a particular brace. However, VAS scores may still be helpful for comfort assessment. Indeed, an objective methodology to characterise wearing comfort over time has yet to be developed.

It is possible to compare the decoupled responses to the levels of action measured by other evaluation tools as presented in two previous studies $[18,19]$. The numerical study highlighted the influence of brace design on its ability to prevent a drawer motion. The presented braces essentially differed in terms of strap and fabric layout and of fabric stiffness. Brace circumferences may also vary: even if sizes were chosen accordingly to the manufacturer's size charts, a product may size too small and another too big. By selecting a factor range representative of the 4 tested braces, the numerical model predicted efficiency indexes ranging from 1.9 to $3.4 \mathrm{~N} / \mathrm{mm}$ and corresponding pressure indexes between 2.9 and $7.1 \mathrm{kPa}$. The exact same braces have also been experimentally tested on the developed surrogate lower limb and the following mean drawer indexes have been measured:

- Brace \#1: $1.85 \pm 0.36 \mathrm{~N} / \mathrm{mm}$.

- Brace \#2: $5.49 \pm 0.69 \mathrm{~N} / \mathrm{mm}$.

- Brace \#3: $3.36 \pm 0.53 \mathrm{~N} / \mathrm{mm}$.

The most efficient brace was found to be the same as in the present study. It is noteworthy that indexes computed from arthrometer measurements are higher than those computed from the data measured by the test machine. Some numerical comparison in this study also suggested that testing these braces on a real limb would yield even lower efficiency indexes, which was found here to be incorrect. This may be partially explained by the fact that in-vivo measurements may be slightly influenced by proprioceptive and active reaction of the subjects. Indeed, the GNRB ${ }^{\circledR}$ arthrometer has been reported to slightly underestimate differential laxities compared to a standard technique [23], in this case 
an intraoperative navigation measurement. This difference might be partially due to involuntary muscle contraction, and to the fact that the anterior motion may be associated to rotation of the joint. The passive state of the limb was indeed subject to caution: several subjects reacted to pain at high loads by contracting hamstrings. Consequently, the measured reaction forces and computed stiffness indexes may be overestimated compared to a complete passive response (e.g. cadaver knee). This assumption is partially confirmed when comparing computed indexes to data available in the literature on cadaver knees: original data from [24] allowed to compute mean $k$ indexes of 10.1 and $2.9 \mathrm{~N} / \mathrm{mm}$ for healthy and pathological knees, and $7.1 \mathrm{~N} / \mathrm{mm}$ for the ACL contribution. Even if the test conditions and specimen were not equivalent, it can reasonably be assumed that there may be non-negligible contribution of active stiffening of the joint in the measures from the GNRB ${ }^{\circledR}$ system. As knee braces have been reported to modify muscle activation of the lower limb [25, 26, 27, 28], the decoupled responses may also involve indirect active stiffening. An interesting addition to this study would have been to reproduce these experiments while measuring the muscle activity using electromyography (agonist/antogonist contraction/co-contraction).

Besides, another potential bias leading to a slight overestimation of the brace effect was that the brace was not entirely free around the joint, as it would be in a natural standing position; the back fabric fitted between the limb and the support of the GNRB ${ }^{\circledR}$.

The effect of helical straps of brace \#2 was also characterised by the test machine; removing this feature resulted in a decrease of the $k$ index by a factor 2. Besides, this feature also had an effect of preventing the drop in reaction force for displacements higher than $8 \mathrm{~mm}$, and the comparison with the present study shows that this feature definitely helped reducing the drawer.

Altogether, the characterised in-vivo efficiencies of these fabric-based braces as measured in this study give more optimistic results than what had been measured using a surrogate limb as testing device. Theses knee braces were found to effectively replace the mechanical role of the ACL up to anterior displacements of 1-4 mm, and even provided a corrected stiffness of $81 \%$ of the healthy knee for the most efficient brace at $5 \mathrm{~mm}$, whereas the relative stiffness of the pathological knee was only $57 \%$. [24] showed that this value of $5 \mathrm{~mm}$ corresponds to the mean anterior displacement at which the mechanical behaviour of the joint changes from a low stiffness region $(7.3 \mathrm{~N} / \mathrm{mm})$ to a high stiffness region $(35.7 \mathrm{~N} / \mathrm{mm})$ because of the tensioning of the ACL. Consequently, the brace was able to compensate for a deficient ACL in the low stiffness region only. This is in agreement with the literature [29]. More efficient brace designs should ideally mimic the non-linear mechanical behaviour of the ligament and induce an increase of the stiffening level as the anterior displacement increases, instead of quickly reaching a maximal load as seen in Figure 7. This can be done by operating on both passive and active mechanisms. The most straightforward technique is to optimize brace designs in order to maximize their passive stiffness, by using standardised surrogate limbs as testing devices. However, joint stability also comes from muscle activation. [30] used an arthrometer 
to measure sagittal-plane shear stiffness of passive and active knees. Passive knees exhibited a mean stiffness of $18.7 \mathrm{~N} / \mathrm{mm}$ (men) and $19.3 \mathrm{~N} / \mathrm{mm}$ (women) while active limbs resulted in stiffer joints: $70.9 \mathrm{~N} / \mathrm{mm}$ (men) and $40.7 \mathrm{~N} / \mathrm{mm}$ (women). That is, the stabilizing effect of an optimised brace could be enough to compensate for a deficient ACL if the muscles are recruited as the main active stabilizers, as proposed by [28]. Consequently, novel methods have yet to be developed to understand and evaluate the effect of brace design on its ability to stimulate the active recruitment of the musculature to participate in maintaining joint stability. It is also important to note that a high patient-specificity was found in the results, characterised by a large scattering, and the given conclusions are based on mean tendencies. However, the best brace was not always the same: it was brace \#1 in 4 cases, brace \#2 in 7 cases, brace \#3 in 4 cases and the compression sleeve in 1 case. Consequently, a patient-specific approach is strongly desirable when actually selecting a brace for a given subject.

\section{Conclusions}

The ability of three commercial fabric hinged knee braces to effectively compensate for a deficient ACL was tested in-vivo using a GNRB ${ }^{\circledR}$ arthrometer on 16 patients. It was determined that based on subjective feelings, subjects were not able to predict which brace would be the most efficient on themselves, highlighting the importance of objective testing procedures for these devices. Subsequently, it was found that these braces effectively replaced the mechanical role of the ACL for low displacement - low force, i.e. within the low stiffness region of this structure. Finally, some results suggested that braces may stimulate an indirect stiffening mechanism (stabilizing contraction of muscles), which should be further investigated as it might prove to be a major part of their efficiency in active dynamic situations. This study was part of a global approach in evaluating these devices which will hopefully lead to the introduction of efficiency-labels directed at manufacturers, for the benefit of the patients. Further studies should also consider a patient-specific approach.

\section{Acknowledgements}

This work was funded in part by the ANRT (Association Nationale de la Recherche et de la Technologie) and the following orthotic manufacturers: Thuasne ${ }^{\circledR}$, Gibaud ${ }^{\circledR}$ and Lohmann-Rauscher ${ }^{\circledR}$.

[1] S. Bollen, Epidemiology of knee injuries: diagnosis and triage, British journal of sports medicine 34 (3) (2000) 227-228.

[2] M. D. Gordon, M. E. Steiner, Anterior cruciate ligament injuries, Orthopaedic Knowledge Update: Sports Medicine. Rosemont, IL: American Academy of Orthopaedic Surgeons (2004) 169-181. 
[3] K. C. Miyasaka, D. M. Daniel, M. L. Stone, P. Hirshman, The incidence of knee ligament injuries in the general population, Am J Knee Surg 4 (1) (1991) 3-8.

[4] S. Dojcinovic, E. Servien, T. A. S. Selmi, C. Bussière, P. Neyret, Instabilités du genou, EMC - Rhumatologie-Orthopédie 2 (4) (2005) 411-442. doi:10.1016/j.emcrho.2005.04.003.

[5] M. Collette, J. Courville, M. Forton, B. Gagnière, Objective evaluation of anterior knee laxity; comparison of the KT-1000 and GNRB $\AA$ arthrometers, Knee surgery, sports traumatology, arthroscopy: official journal of the ESSKA 20 (11) (2012) 2233-2238. doi:10.1007/s00167-011-1869-2.

[6] D. Daniel, L. Malcom, G. Losse, M. Stone, R. Sachs, R. Burks, Instrumented measurement of anterior laxity of the knee, J Bone Joint Surg Am 67 (5) (1985) 720-726.

[7] H. Robert, S. Nouveau, S. Gageot, B. Gagnière, Nouveau système de mesure des laxités sagittales du genou, le GNRB $\cap$. application aux ruptures complètes et incomplètes du ligament croisé antérieur, Revue de Chirurgie Orthopédique et Traumatologique 95 (3) (2009) 207-213. doi:10.1016/j.rcot.2008.12.007.

[8] S. A. Paluska, D. B. McKeag, Knee braces: current evidence and clinical recommendations for their use, American Family Physician 61 (2) (2000) 411-418, 423-424.

[9] P. Thoumie, P. Sautreuil, E. Mevellec, Orthèses de genou. première partie : Évaluation des propriétés physiologiques à partir d'une revue de la littérature. knee orthosis. first part : evaluation of physiological justifications from a literature review, Annales de Réadaptation et de Médecine Physique 44 (9) (2001) 567-580. doi:10.1016/S0168-6054(01)00157-X.

[10] P. Thoumie, P. Sautreuil, E. Mevellec, Orthèses de genou. Évaluation de l'efficacité clinique à partir d'une revue de la littérature. knee orthosis., Annales de Réadaptation et de Médecine Physique 45 (1) (2002) 1-11. doi:10.1016/S0168-6054(01)00166-0.

[11] M. Genty, C. Jardin, Place des orthèses en pathologie ligamentaire du genou. revue de la littérature, Annales de Réadaptation et de Médecine Physique 47 (6) (2004) 324-333. doi:10.1016/j.annrmp.2004.05.001.

[12] J. Beaudreuil, S. Bendaya, M. Faucher, E. Coudeyre, P. Ribinik, M. Revel, F. Rannou, Clinical practice guidelines for rest orthosis, knee sleeves, and unloading knee braces in knee osteoarthritis, Joint Bone Spine 76 (6) (2009) 629-636. doi:10.1016/j.jbspin.2009.02.002.

[13] K. T. L. Chew, H. L. Lew, E. Date, M. Fredericson, Current evidence and clinical applications of therapeutic knee braces, American 
Journal of Physical Medicine \& Rehabilitation 86 (8) (2007) 678-686. doi:10.1097/PHM.0b013e318114e416.

[14] P. Ribinik, M. Genty, P. Calmels, Évaluation des orthèses de genou et de cheville en pathologie de l'appareil locomoteur. avis d'experts, Journal de Traumatologie du Sport 27 (3) (2010) 121-127. doi:10.1016/j.jts.2010.07.008.

[15] C. Beck, D. Drez, J. Young, W. D. Cannon, M. L. Stone, Instrumented testing of functional knee braces, The American Journal of Sports Medicine 14 (4) (1986) 253-256. doi:10.1177/036354658601400401.

[16] P. W. Cawley, E. P. France, L. E. Paulos, Comparison of rehabilitative knee braces, The American Journal of Sports Medicine 17 (2) (1989) 141 -146. doi:10.1177/036354658901700201.

[17] S. H. Liu, T. Lunsford, S. Gude, J. Vangsness, C T, Comparison of functional knee braces for control of anterior tibial displacement, Clinical orthopaedics and related research 303 (1994) 203-210.

[18] B. Pierrat, J. Molimard, L. Navarro, S. Avril, P. Calmels, Evaluation of the mechanical efficiency of knee orthoses: a combined experimental-numerical approach, Proceedings of the Institution of Mechanical Engineers, Part H: Journal of Engineering in MedicineIn press.

[19] B. Pierrat, J. Molimard, L. Navarro, S. Avril, P. Calmels, Evaluation of the mechanical efficiency of knee braces based on computational modelling, Computer Methods in Biomechanics and Biomedical Engineering.

[20] N. Lefevre, Y. Bohu, J. F. Naouri, S. Klouche, S. Herman, Validity of GNRB(囚) arthrometer compared to telos ${ }^{\mathrm{TM}}$ in the assessment of partial anterior cruciate ligament tears, Knee surgery, sports traumatology, arthroscopy: official journal of the ESSKAdoi:10.1007/s00167-013-2384-4.

[21] C. Bonifasi-Lista, S. P. Lake, M. S. Small, J. A. Weiss, Viscoelastic properties of the human medial collateral ligament under longitudinal, transverse and shear loading, Journal of orthopaedic research: official publication of the Orthopaedic Research Society 23 (1) (2005) 67-76. doi:10.1016/j.orthres.2004.06.002.

[22] K. M. Quapp, J. A. Weiss, Material characterization of human medial collateral ligament, Journal of biomechanical engineering 120 (6) (1998) 757-763.

[23] J.-Y. Jenny, J. Arndt, Mesure de la laxité antérieure du genou par des radiographies dynamiques et le système GNRBß. comparaison avec la mesure naviguée peropératoire, Revue de Chirurgie Orthopédique et Traumatologique 99 (6, Supplement) (2013) S201-S204. doi:10.1016/j.rcot.2013.07.007. 
[24] P. Eagar, M. L. Hull, S. M. Howell, A method for quantifying the anterior load-displacement behavior of the human knee in both the low and high stiffness regions, Journal of Biomechanics 34 (12) (2001) 1655-1660. doi:10.1016/S0021-9290(01)00142-7.

[25] L. R. Osternig, R. N. Robertson, Effects of prophylactic knee bracing on lower extremity joint position and muscle activation during running, The American Journal of Sports Medicine 21 (5) (1993) 733-737. doi: $10.1177 / 036354659302100518$.

[26] D. K. Ramsey, P. F. Wretenberg, M. Lamontagne, G. Németh, Electromyographic and biomechanic analysis of anterior cruciate ligament deficiency and functional knee bracing, Clinical Biomechanics 18 (1) (2003) 28-34. doi:10.1016/S0268-0033(02)00138-9.

[27] D. Théoret, M. Lamontagne, Study on three-dimensional kinematics and electromyography of ACL deficient knee participants wearing a functional knee brace during running, Knee Surgery, Sports Traumatology, Arthroscopy 14 (6) (2006) 555-563. doi:10.1007/s00167-006-0072-3.

[28] E. M. Wojtys, S. U. Kothari, L. J. Huston, Anterior cruciate ligament functional brace use in sports, The American Journal of Sports Medicine 24 (4) (1996) 539-546. doi:10.1177/036354659602400421.

[29] T. Branch, R. Hunter, P. Reynolds, Controlling anterior tibial displacement under static load: a comparison of two braces, Orthopedics 11 (9) (1988) 1249-1252.

[30] E. M. Wojtys, J. A. Ashton-Miller, L. J. Huston, A gender-related difference in the contribution of the knee musculature to sagittal-plane shear stiffness in subjects with similar knee laxity, The Journal of bone and joint surgery. American volume 84-A (1) (2002) 10-16. 